\title{
Optimistic Medium Access Control using Gait Anaysis in Body Sensor Networks
}

\author{
Tiong Hoo Lim \\ Electrical and Electronic Engineering \\ Institut Teknologi Brunei, \\ Gadong, BE3119 \\ Brunei Darussalam \\ Email: lim.tiong.hoo@itb.edu.bn
}

\author{
Teng Weng \\ Department of Computer Science \\ University of York, York \\ North Yorkshire, YO10 5GH \\ United Kingdom \\ Email: t1540@york.ac.uk
}

\author{
Iain Bate \\ Department of Computer Science \\ University of York, York \\ North Yorkshire, YO10 5GH \\ United Kingdom \\ Email: iain.bate@york.ac.uk
}

\begin{abstract}
Body Sensor Networks (BSNs) have been used to provide continuous remote health monitoring and analysis of physiological parameter of patient. These devices can be attached to different parts of the human body to capture and wirelessly transmit health statistics in a wearable, non-invasive form factor. However, the small physical size of the sensor node used in BSNs can result in irregular transmission failures caused by body shadowing. Body shadowing can disrupt the radio communication due to body movement preventing the radio signal from passing through. In this work, an innovative approach based on body positioning prediction is applied to minimise the transmission failures and lower the power consumption. By analysing the impact of different leg positions and twiddling of the radio signal, an algorithm to adapt the periodicity of the transmission period for reliable transmission is proposed. The results from hardware experiment have shown that the proposed solution can achieve transmission success rate above $90 \%$ with reducing the energy consumption by about $50 \%$.
\end{abstract}

Keywords-Body Sensor Networks, Gait Analysis, Body Shadowing, MAC, Duty Cycle;

\section{INTRODUCTION}

Wireless Sensor Networks (WSNs) have been applied to a range of domains including environments monitoring, factory automation and home monitoring system. Among them, Body Sensor Network (BSN) provides a novel uses in health-care especially in the health monitoring field. The abilities of BSN to provide continuous remote health care monitoring have resulted in various medical applications. A BSN consists of multiple wireless sensor nodes placed on or implanted into the human body. These nodes contain a wireless transceiver, memory storage, battery and one or more physio-sensors that measure biometrical properties such as heart rate and ECG. The nodes can be placed at the different parts of the body and can work individually or cooperate with each other in providing sensing, processing, and communication capabilities. Through the wireless transmission, the biometrical properties are gathered by an aggregator node, such as a smart phone, which acts as a sink. Once the data are collected, the sink will send the information to the global base station for proper health checking.

To reduce disruption to the patient daily activities, the size of the BSN node needs to be minimised [1]. As a result, the small physical size can lead to the shortage of energy storage [1]. In BSN, the energy is utilised in three domains namely data sensing, data communication and data processing. The power used for data communication is usually more than data communication as specified in Chipcon datasheet [2]. According to [3], energy consumption during the Clear Channel Access (CCA) $\left(E_{C C A}\right)$ is $0.18 \mathrm{~mJ}$ while data receiving $\left(E_{\text {rec }}\right)$ and sending $\left(E_{\text {sent }}\right)$ are both $0.12 \mathrm{~mJ}$ for 1 Byte of data. This number is significantly higher than the processing of 1 instruction $(0.2 \mathrm{~nJ})$. Furthermore, communication failure is more common in BSN than traditional wireless. The energy consumption during interference is usually higher due to retransmission [4]. If communication failures can be predicted, a node can delay or adapt its transmission.

Many researchers have engaged in various adaptive techniques to control its Medium Access Control (MAC) or routing protocol [4]. The objective of this paper is to reduce the transmission failures caused by the movement of the human body that can restrict the radio transmission and interfere with the data transmission. An Optimistic Medium Access Control Algorithm (OMAC) is proposed by predicting and determining the body position for the best fit for wireless transmission. The motivation behind this paper is that the human body is moving all the time. By locating the non-obstructive body position for transmission and adapting the radio signal strength in the MAC can improve the rate of a successfully transmission and reduce the energy consumption. The movement pattern can be predicted using gait kinematics especially when someone is walking at a constant pace [5]. We believe this field has not been successfully being implemented in real hardware and a promising result has been shown in this paper for the first time.

This paper begins by highlighting works related to reduce the energy consumption in BSN in Section II. The design of the OMAC is presented in Section III. An analysis of radio signal strength to determine the minimum transmission power and legs position for reliable transmission is presented in Section IV. The relationship between different leg positions and the accelerator value is studied in Section V. The OMAC is tested and evaluated experimentally using Telosb motes in Section VI. The results are discussed in Section VII before we conclude the paper in Section VIII.

\section{RELATED WORKS}

The sensor placements are restricted to the subject's body parts and usually attached on the wrist and ankle. In order 
to achieve maximum receiving rate, the attachment location of these sensor node is critical. The most common locations are waist, wrist, thigh, ankle and head. Some studies have evaluated the effectiveness of different attaching locations. Bourke et al. [6] have used the accelerometer sensors mounted on the trunk and thigh to detect fall and suggested that the trunk was the optimum location for a fall sensor. Kangas et al. [7] have shown that the best sensor location were the waist and head where the head level had the highest accuracy. However, it can be inconvenient and impractical for the users to wear the sensor on their head all the time. As a result, an accelerometer sensor node worn on the waist might be the best choice.

In [8], Qi et al. analyzed the impact of different sensor positions and body movements on the radio signal by measuring the Received Signal Strength Indication (RSSI) and the packet delivery rate (PDR). They have shown that the radio signal can be affected by the hand and leg movement and the RSSI deviation varied depending on the placement of the sensors. Hence, there is a need to determine the highest radio signal for transmission

Ye et al. [9] attempts to reduce energy consumption by duty-cycling the radio's sleep pattern of the nodes inside a cluster. The proposed MAC protocol reduces channel contention between nodes but does not prevent communication failure due to interference or obstruction. There is a need to predict the body movement and adapt the sleep pattern in MAC accordingly to reduce failure rate.

\section{The Optimistic Medium AcCess Control ALGORITHM}

In this section, we present the OMAC, an algorithm that uses the accelerator reading to determine the best leg position for successful communication. Using accelerator data to determine different position and trajectory, an algorithm to adjust the duty cycle of the node is designed. The OMAC consists of three main hardware as shown in Figure 1 namely: the aggregation node attached on the waist for the best receiving position [7], a movement sensing node attached to ankle of the user's body which is the critical location for activity recognition [10] and a base station that will connected to a laptop through the USB interface.

\section{A. Algorithm Design}

In order to ensure that data to be delivered in a reliable way, it is necessary to determine the best time for transmission when non-obstructive communication is available. This can be achieved using the on-board accelerator. Previous studies have shown that it is possible to use the reading of the accelerator to determine the body position [6], [8]. The flow diagram for the OMAC is shown in Figure 2.

Gait analysis is performed using the accelerator reading taken from the sensor node [11]. When the system starts, the timer is initialised. A counter (C) is used to capture the number of sensor reading stored in the node for every $50 \mathrm{~ms}$. It should be noted that the sensing data will not be deleted until it has been successfully delivered. Each TelosB node can only store up to 5000 sensor readings due to the limited memory size $(10 \mathrm{kB})$. To ensure the memory buffer does not overflow, a maximum value of 1000 readings is assigned in the algorithm

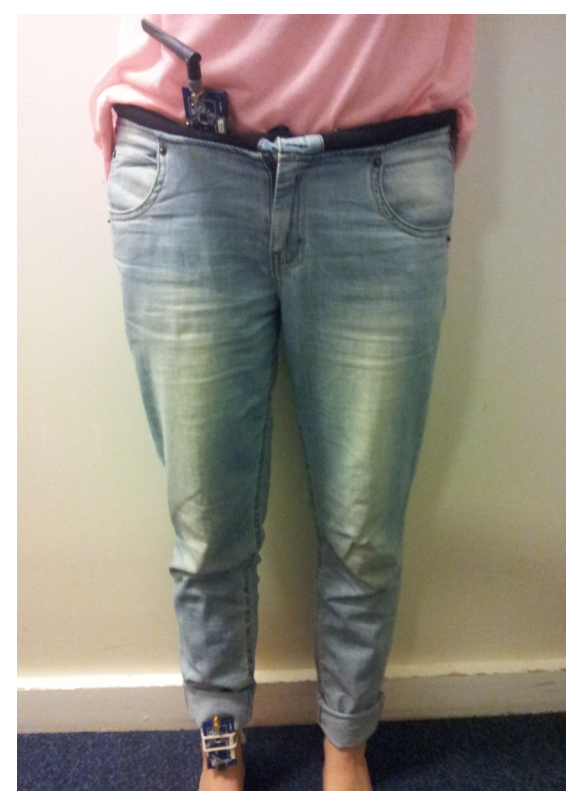

Fig. 1. The placement of the transmitter (Leg) and the receiver (waist).

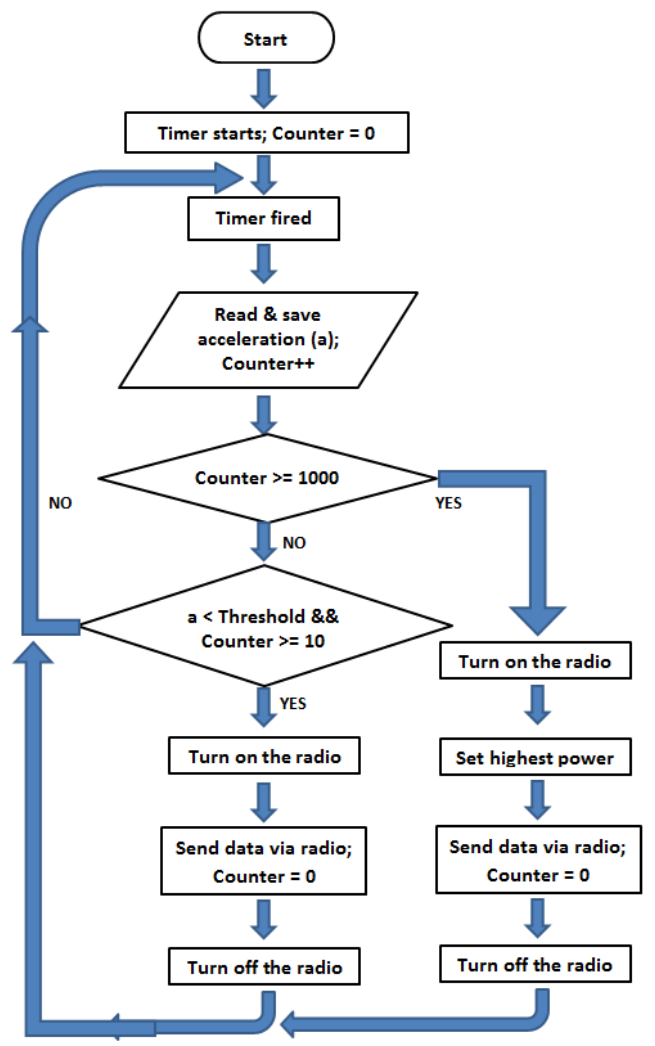

Fig. 2. The radio is turned off when the counter is less than 1000 or when accelerator reading is less than threshold $a$. The radio is only turn on and the packets are transmitted when the accelerator reading ge $a$.

for this experiment. When 1000 readings are collected, all the packets stored in the buffer will be transmitted using the maximum transmission power $(-25 \mathrm{~dB})$.

In our work, we set the minimum value of $C_{\min }=10$ and $C_{\max }=1000$. When $\mathrm{C}<1000$ and the leg is not in the best position to transmit the packet with minimum power, 
the radio transceiver will be switched off to conserve energy. The radio transceiver is only turned on when the gait analysis algorithm has detected the reading on the accelerator is above the threshold $(a) . C_{\min }$ is set to 10 to ensure that the radio is only turned on when there are sufficient numbers of readings in the buffer to reduce low data transmission. An analysis has shown that it takes $1 \mathrm{sec}$ for human to finish a gait cycle. During a gait cycle, the sensor will capture 20 readings. If the two conditions are both satisfied, the radio transceiver is turned on for transmission. If not, the system will wait for next cycle. There two values can futher be optimised in future work.

\section{ANALYSING THE DIFFERENT BODY POSITION TO DETERMINE THE TRANSMISSION POWER}

In order for the algorithm to function, it is necessary to analyze how the wireless radio signal strength changes with different leg positions. This can be achieved by computing the number of packets received by a node attached on the waist of the person walking naturally. It is also necessary to determine the minimum power signal required to transmit the packet from a node attached to the ankle. The results from experiments will determine the minimum power setting and the threshold value of $a$.

\section{A. Experimental Setup}

The radio node used in this experiment is Telosb mote module with CC2420 Radio Frequency (RF) transceiver using the configuration shown in Table I [12]. To obtain valuable results, one radio node is placed on the ankle and one receiver node is placed on front waist with the antenna as displayed in Figure 1.

TABLE I. TINYOS CONFIGURATIONS
\begin{tabular}{|c|c|}
\hline Parameters & Values \\
\hline Tx interval: & $250 \mathrm{~ms}$ \\
\hline Tx Channel: & 26 \\
\hline MAC: & 802.15 .4 (CSMA/CA) \\
\hline
\end{tabular}

In each experiment, 500 packets are transmitted using 8 transmission power levels $\left(T x_{p w}\right)$ : $-25 \mathrm{dBm},-15 \mathrm{dBm},-10 \mathrm{dBm}$, $-7 \mathrm{dBm},-5 \mathrm{dBm},-3 \mathrm{dBm},-1 \mathrm{dBm}$, and $0 \mathrm{dBm}$. The receiver records the RSSI, the Radio Signal Strength Indication, of received packets and computes the number of packets received. The RSSI from three leg positions: (1) backward; (2) midswing; (3) forward are recorded from three different persons, two males and one female.

\section{B. Results}

Figure 3 shows the RSSI values for the three different leg positions. From the chart, it can be seen that there is a strong correlation between the leg position and RSSI. The RSSI value in the forward position is the highest, which is followed by the value in the backward position. The RSSI value is the lowest when the leg is in the mid swing position. In the mid-swing position, the transmission path is heavily blocked. Therefore, the attenuation is the largest among the three positions. Likewise, in the backward position, the blocking extent is lower than the mid-swing position thus the attenuation is smaller.

The experimental results also show the PRR in the midswing position decreases at $T x_{p w}=-7 \mathrm{dbm}$ and drops to 0 at

\section{PRR vs Power}

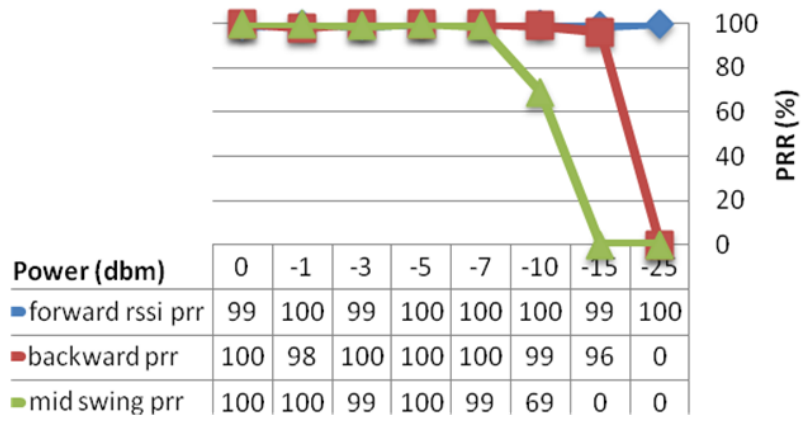

Fig. 3. The forward position has the highest Packet Receive Rate and required lower $T x_{p w}(-25 \mathrm{~dB})$ compared to backward and mid-swing position.

$T x_{p w}=-15 \mathrm{dbm}$. The PRR of the backward position goes down slowly at $T x_{p w}=-15 \mathrm{dbm}$ and reaches 0 at $T x_{p w}=-25 \mathrm{dbm}$. The forward position only requires $T x_{p w}=-25 \mathrm{dBm}$ power to ensure packet delivery. Hence, in the next experiment, $T x_{p w}=-25 \mathrm{dBm}$ is configured to evaluate the algorithm.

\section{USING GAIT ANALYSIS TO DETERMINE FORWARD LEG POSITION THRESHOLD}

In this section, an experiment to detect the forward leg position using the sensor acceleration data is presented.

\section{A. Experimental Setup}

In this experiment, two-axis accelerometer (MTS-3000) and Telosb mote module (CM3000) are used. The accelerometer is placed on the subjects ankle to sense the acceleration in two directions, $\mathrm{x}$-axis and $\mathrm{y}$-axis. It should be noted that only x-axial (backward - forward) acceleration readings are recorded as the main movement of the ankle is on the y-axis. Three healthy subjects (two males, one female) are asked to walk naturally along in the hallway, wearing their own flat shoes and without receiving any special instruction. They walk about $30 \mathrm{~m}$ at their normal speed. Each experiment is repeated. 13 acceleration thresholds are selected based on the maximum and minimum value of the accelerometer reading: 2600, 2610, 2620, 2630, 2640, 2650, 2660, 2670, 2680, 2690, 2700, 2710 and 2720 .

\section{B. Evaluation Metrics}

To determine the accuracy of the accelerator threshold to detect the forward position, the following metrics are computed:

$$
\begin{aligned}
& \text { Sensitivity }=\frac{(\text { True Positive })}{(\text { True Positive }+ \text { False Negative })} \\
& \text { Specificity }=\frac{(\text { True Negative })}{(\text { True Negative }+ \text { False Positive })}
\end{aligned}
$$




\section{Results}

The graph of sensitivity against specificity is shown in Figure 4 . When the threshold is set to 2670 , the sensitivity is about 0.88 and the specificity is around 0.92 , which means about $88 \%$ true positive and $92 \%$ true negative have been detected. Moreover, from the graph, it can be seen the area under the curve is large area. This means the gait analysis can be used to detect forward leg position.

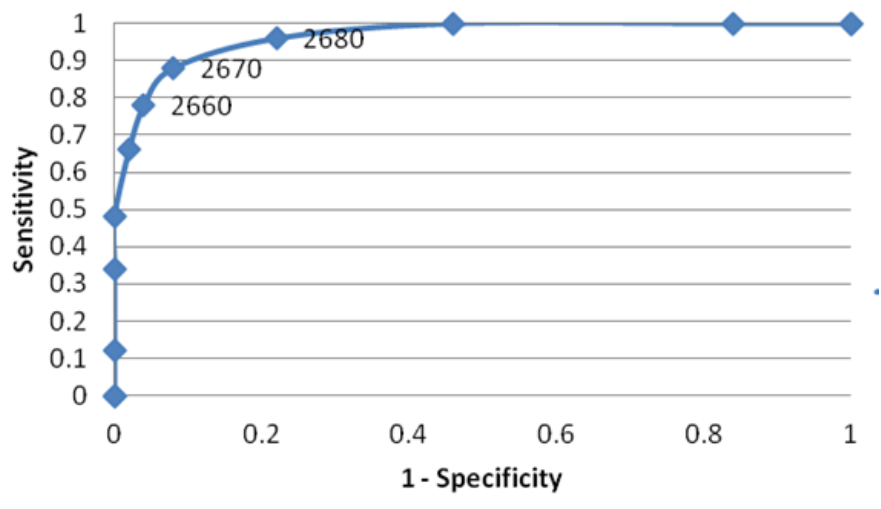

Fig. 4. The sensitivity and specificity of different acceleration threshold where the $88 \%$ sensitivity can be achieved when $a=2670$.

\section{EVAlUATION OF THE OPPORTUNISTIC MAC ALGORITHM}

To evaluate the effectiveness of the OMAC, one healthy subject walks naturally along in the hallway, wearing a flat shoe without receiving any special instruction. The sensor node is placed on the healthy subject's ankle to transmit 100 data packets, each with 20 Bytes of information to the receiver every $1 \mathrm{sec}$. If the transmission fails, the packet will be retransmitted until it is delivered successfully.

The experiments are conducted under 4 conditions:

1. OMAC, $T x=-25 \mathrm{dBm}$.

2. Without OMAC, $T x=-25 d B m$.

3. Without OMAC, $T x=-15 d B m$.

4. Without OMAC, $T x=-7 \mathrm{dBm}$.

To measure reliability, the PDR and the transmission time are calculated using the approaches in [13]. Using the value discussed in Section I, the theoretical energy consumption is calculated using the equation [3]:

$$
E_{\text {used }}=E_{C C A}+E_{\text {sent }}+E_{\text {rec }}
$$

\section{RESULTS AND DiscUSSIONS}

Table II shows the results between transmission with and without the OMAC in term of PDR rate and energy consumption. With OMAC, the PDR is $92 \%$ when $T x_{p w}=-25 \mathrm{dBm}$, and consumes less energy $(289 \mathrm{~mJ})$ compared to transmission without OMAC. The sensor nodes without OMAC have relatively poorer performance. Although without OMAC at the $T x_{p w}=-7 \mathrm{dBm}$ can guarantee no packet lost, the energy is consumed highest $(583.4 \mathrm{~mJ})$. When the $T x_{p w}=-25 \mathrm{dBm}$, the PDR $=32 \%$ but consumed $522.4 \mathrm{~mJ}$ energy and took $45.25 \mathrm{~s}$ to transmit the packets. With $T x_{p w}=-15 \mathrm{dBm}$, the PDR $=69 \%$ and consumes $407.1 \mathrm{~mJ}$ energy for $18.13 \mathrm{~s}$. Based on the above,
TABLE II. RESULTS SHOWING THE SUCCESS RATE AND ENERGY CONSUMPTION WITH AND WITHOUT OMAC

\begin{tabular}{llll}
\hline Algorithm & $\begin{array}{l}\text { PDR } \\
(\%)\end{array}$ & Time(s) & $\begin{array}{l}E_{u s e d} \\
(\mathrm{~mJ})\end{array}$ \\
\hline OMAC & 92 & 14 & 289 \\
$\left(T x_{p w}=-25 d B m\right)$ & & & \\
Non-OMAC $\left(T x_{p w}=-25 d B m\right)$ & 32 & 45.25 & 522.4 \\
Non-OMAC $\left(T x_{p w}=-15 d B m\right)$ & 69 & 18.13 & 407.1 \\
Non-OMAC $\left(T x_{p w}=-7 d B m\right)$ & 100 & 12.5 & 583.4 \\
\hline
\end{tabular}

it can be concluded the sensor node is more energy efficiently using OMAC. However, the test data and result samples are small. More experiments using a systematic approach such as in [13] are required as future work to ensure the results obtained are valid and of good quality.

\section{CONCLUSION}

Using Gait analysis to determine position and the transmission trajectory, we have proposed the OMAC that adjusts the transmission signal strength according to the body position. Experimental results have shown that OMAC can achieve a higher PDR with a lower energy consumption compare to normal transmission without OMAC. However, further experimens using a systematic approach is necessary to validate the results and verify that the algorithms will work with different body sizes and ages.

\section{REFERENCES}

[1] B. Lo and G. Yang, "Key technical challenges and current implementations of body sensor networks," in 2nd International Workshop on Body Sensor Networks, 2005.

[2] CC2420 2.4 GHz IEEE 802.15. 4/ZigBee-ready RF Transceiver Data Sheet (rev. 1.3), Chipcon, A. S., 2011, rev. 3.

[3] M. Amiri, "Measurements of energy consumption and execution time of different operations on Tmote sky sensor nodes," Ph.D. dissertation, Masaryk University, 2010.

[4] T. H. Lim, I. Bate, and J. Timmis, "A self-adaptive fault-tolerant systems for a dependable wireless sensor networks," Design Automation for Embedded Systems, pp. 1-28, 2014.

[5] D. Gafurov, E. Snekkenes, and P. Bours, "Gait authentication and identification using wearable accelerometer sensor," in IEEE Workshop on Automatic Identification Advanced Technologies, 2007, pp. 220-225.

[6] A. Bourke, J. O' brien, and G. Lyons, "Evaluation of a threshold-based tri-axial accelerometer fall detection algorithm," Gait \& posture, vol. 26, no. 2, pp. 194-199, 2007.

[7] M. Kangas, A. Konttila, P. Lindgren, I. Winblad, and T. Jamsa, "Comparison of low-complexity fall detection algorithms for body attached accelerometers," Gait \& posture, vol. 28, no. 2, pp. 285-291, 2008.

[8] X. Qi, G. Zhou, Y. Li, and G. Peng, "RadioSense: Exploiting wireless communication patterns for body sensor network activity recognition," in RTSS, 2012, pp. 95-104.

[9] W. Ye, J. Heidemann, and D. Estrin, "An energy-efficient MAC protocol for wireless sensor networks," in 21st Annual Joint Conference of the IEEE Computer and Communications Societies, vol. 3. IEEE, 2002, pp. 1567-1576.

[10] M. Keally, G. Zhou, G. Xing, J. Wu, and A. Pyles, "Pbn: towards practical activity recognition using smartphone-based body sensor networks," in the 9th ACM Conference on Embedded Networked Sensor Systems. ACM, 2011, pp. 246-259.

[11] M. W. Whittle, Gait analysis: an introduction, 2003.

[12] (2011) Memsic: Telosb mote. [Online]. Available: http://www.memsic.com/products/wireless-sensor-networks.html

[13] T. H. Lim, I. Bate, and J. Timmis, "Validation of performance data using experimental verification process in wireless sensor network," in IEEE 17th Conference on Emerging Technologies Factory Automation, 2012, pp. 1-8. 